\title{
Human settlement and infrastructure monitoring with hyperspectral imaging
}

\author{
Andrea Marinoni \\ Centre for Integrated Remote Sensing and \\ Forecasting for Arctic Operations (CIRFA) \\ Dept. of Physics and Technology \\ UiT - the Arctic University of Norway \\ Tromsø, Norway \\ andrea.marinoni-at-uit.no
}

\author{
Uta Heiden \\ German Remote Sensing Data Center \\ Deutsches Zentrum für Luft- und Raumfahrt \\ $D L R$ \\ Oberpfaffenhofen, Germany \\ uta.heiden-a-dlr.de
}

\author{
Paolo Gamba \\ Dept. of Electrical, Biomedical \\ and Computer Engineering \\ University of Pavia \\ Pavia, Italy \\ gamba-at-unipv.it
}

\begin{abstract}
This paper reviews in detail the contributions of hyperspectral imaging to the topic of urban remote sensing. Hyperspectral imaging is traditionally connected to the spectral characterization of surface materials. Moreover, urban areas are characterized by a very complex geometrical structure, which requires either very high spatial resolution or complex unmixing procedures based on linear and non-linear mixing models. Nonlinear unmixing and material mapping using both spectral and spatial features are therefore two important topics when using hyperspectral imaging to monitor human settlements and infrastructures. Finally, even when no specific material and or urban element is sought, the mixture of artificial (as opposed to natural) materials in human settlements can be used to delineate their extents, with excellent results with respect to those obtained by multispectral optical sensors with the same spatial resolution.
\end{abstract}

Index Terms-urban unmixing, urban extent extraction.

\section{INTRODUCTION}

$\mathrm{T}$ HE use of hyperspectral imaging to characterize human settlements and artificial infrastructures is less common in technical literature than its counterpart, i.e. the exploitation of hyperspectral data to analyze natural elements such as vegetation, water and soil. As a matter of fact, the main limit to the use of hyperspectral imagers in urban remote sensing application is the (relatively) coarse spatial resolution of existing and planned spaceborne platforms, as well as the high acquisition costs per square $\mathrm{km}$ of airborne platforms. Notwithstanding these limits, there has been a number of works devoted to the use of hyperspectral imaging in urban areas, notably [1] where possible applications in urban areas of the EnMAP sensor have been introduced.

Hyperspectral imaging can be used to monitor human settlements and artificial infrastructure in many ways. The spectral (ultra)fine resolution of these sensors allows the detection and classification of artificial materials [2], as well as their aging situation [3], which are invaluable for the safety of human beings [4]. They are also useful to characterize urban vegetation [5], quickly discriminating between healthy trees and stressed ones. Finally, exploiting spectral and spatial properties of the elements of a scene is the usual way to translate urban hyperspectral images into thematic maps [6,7], for land cover/land use purposes or climate analyses [8].

Since urban details are often very fine, and do not match the spatial resolution of hyperspectral sensors, limited by the large number of bands and the minimum SNR to be guaranteed, material abundances are achieved by means of unmixing techniques [9]. However, the complex interactions due to the urban geometry justify (better, require) the use of non-linear mixing models [10]. By retrieving abundances, more accurate maps as well as biophysical parameters can be extracted. Whenever useful, abundances can be also combined to extract specific targets: this is the case for instance for human settlement extents without any limitation as for the geographical area and the time of acquisition [11].

Following to this quick list of urban remote sensing applications of hyperspectral imaging, Section II is devoted to summarize and discuss existing studies on material and vegetation characterization in urban areas through hyperspectral imaging. Section III introduces the issues related to the two- and three-dimensional landscape of human settlements, and summarizes the approaches designed to tackle with mapping urban elements jointly considering spectral and spatial pattern similarity. Section IV delineates options to exploit spaceborne hyperspectral sensors to extract urban extents at the global level. Finally, Section V includes open challenges and opportunities to be considered for future researches involving hyperspectral imaging of urban areas.

\section{URBAN MATERIAL AND VEGETATION CHARACTERIZATION}

The urban environment is the most complex and heterogeneous landscape on Earth. It is characterized by a frequent change of artificial as well as natural surfaces. Consequently, this leads to a very high spectral and spatial information content of urban hyperspectral imageries and requires (1) a profound knowledge of the chemical composition, physical structure, spectral behavior of urban surfaces, (2) image based effects and (3) specific techniques and analyses concepts for an area-wide surface inventory.

Because of the wide range of thematic applications, several classification schemes have been developed [9, 12, 13]. Most of them can be traced back to the land cover/land use classification scheme of [14], and are the link between the physical surface material characteristics and their functions in the urban ecosystem.

\section{A. Artificial materials}

First basic studies have shown that artificial urban surfaces 
are characterized by material specific reflectance features $[15$,

$13,12]$. Moreover, urban surface material classes are also characterized by high within-class variability as shown in Fig.

1 , which is due to several factors, such as color, coating, degradation [3] and illumination [16]. The highest inter- and intra-class variability can be found for roof materials due to their often varying orientations towards the sun/illumination and the sensor. [17] and [18] have analyzed the bidirectional reflectance behavior of selected roofing materials and found not only variations in amplitude as for most of the materials but also non-systematic hot spot effects especially for metals.

Despite the described spectral complexity in airborne and thus also in spaceborne hyperspectral imageries, there are spectral reflectance characteristics that can be used to identify material type of surfaces. These so called robust spectral features are observable independent from intra-class variability and effects occurring due to preprocessing [13]. Importantly, not only one spectral feature such as the position and depth of a absorption is necessary, the combination of different features is highly recommended and leads to the best separability. Additional height and thermal information can greatly improve the separability [2, 19, 9], especially for materials that are manufactured using the same base material such as roofing tiles and red loose chippings or bitumen and asphalt streets. The correct separation of these surfaces is important because of their different ecological functions in the urban systems.

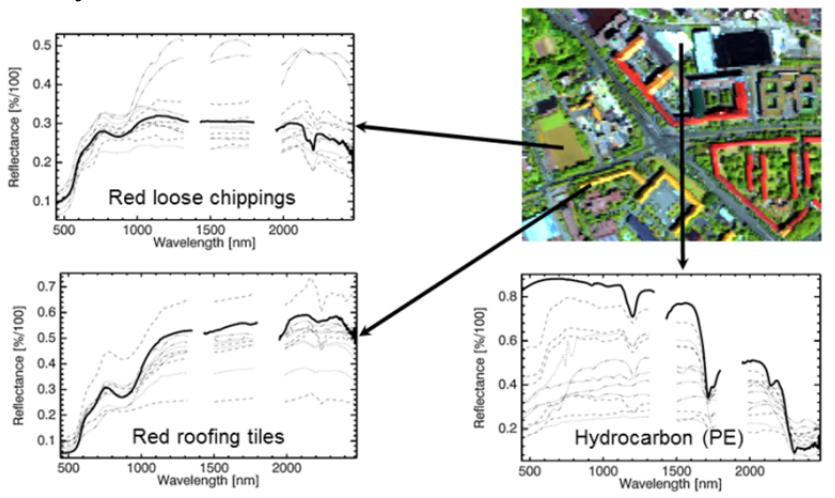

Fig. 1: Spectral separability of urban surface materials based on airborne hyperspectral HyMap image of Munich, Germany (RGB: [1652, 719, 543] $\mathrm{nm})$.

\section{B. Urban vegetation}

Vegetation is especially important in the urban environments due to their positive impacts on urban heat island mitigation, air pollution removal, carbon storage, building energy-use modification, and storm water runoff reduction [20-22].

Tree species mapping is important because limited tree diversity in urban areas increases the likelihood of mass mortality from outbreaks of insects and disease [23]. [24] use airborne hyperspectral AVIRIS data for tree species mapping of the Santa Barbara region, USA, and in [25] balloon-borne hyperspectral imagery is analyzed using classification and unmixing techniques to separate plant species in an urban estuary. The synergistic use of hyperspectral and LIDAR data is well known and can greatly improve results by using both, functional traits from hyperspectral data and structural traits from LIDAR data $[5,26]$. In lower resolution data where crowns can't be resolved, gradients of forest composition have been explored [27]. Results contributed to a better understanding of forest functioning in urban ecosystems and highlight the potential of spaceborne imaging spectrometers such as HyspIRI, PRISMA and EnMAP.

Imaging spectroscopy analyses can also be used to measure air pollution effects on vegetation. [28] have detected different levels of air pollution based on leaf-level reflectance studies of C. betulus. [29] could distinguish trees with lowered chlorophyll content due to a suboptimal habitat quality (more exposure to air pollution) from healthier ones based on a spectral NAOC (Normalized Area Over reflectance Curve). The authors of [30] investigated the effect of ambient air pollution on leafs using high spectral resolution solar-induced steady-state Chl fluorescence. They found an improved ability to detect stressed leafs using this new technique.

\section{MAPPING THE URBAN "BuILTSCAPE"}

As mentioned in the introduction, often the spatial resolution of hyperspectral sensors does not match the size of urban objects. This is definitely true for spaceborne missions, whose spatial resolution is always between 20 and $30 \mathrm{~m}$, because of SNR constraints. Accordingly, most of the pixels in urban areas are mixed ones, and unmixing becomes necessary to extract abundances of artificial/natural materials and eventually mapping biophysical and land use/socio-economic variables. Linear mixture modeling has been widely used in technical literature, especially considering Multiple Endmember Spectral Mixture Analysis (MESMA) [31-33]. This approach has been limited, however, to multispectral (e.g., Landsat) data, where the small amount of bands and the coarse spectral information does not allow a precise characterization of the mixtures. In hyperspectral imaging it is instead natural to move from MESMA to non-linear mixtures, since the data allows detecting much finer spectral details.

Indeed, the extremely complex 2D and 3D patterns of urban elements call for non-linear models to understand the multiple reflections and interactions that concur to the final reflected signal at each wavelength. Accordingly, non-linear unmixing has been introduced in urban remote sensing [10], and different models has been validated and compared. As "nonlinearity" has quite a broad definition, the degree of nonlinearity [34] is also object of investigations, in order to adapt the model to the urban landscape.

The identification and linear and nonlinear combination of endmembers in natural environments has lead to models that have been also tested in urban areas. Since in all these cases

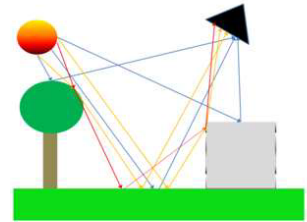

(a)

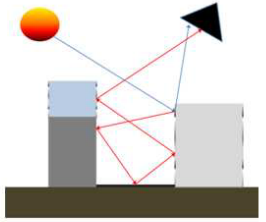

(b)

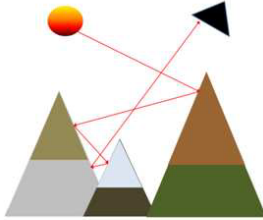

(c)
Fig. 2: Examples of linear and non-linear material interactions adding up, for instance, to non-linear mixing in urban areas. 
bilinear models (such as [35]) explain only a tiny fraction of the mixtures, several models have been proposed to address the issue to track the physical interferences, scatterings and reflections that may occur in geometrically complex mixtures [36, 40]. Following the same line, in [37] a multilinear mixture model including all orders of interactions is used to describe the reflectance of hyperspectral images. Alternatively, the methods introduced in [38] and [39] can be used to retrieve a feasible description of the effects resulting from additive terms assumed to corrupt an originally linear mixture term.

Several papers have recently addressed the topic of nonlinearity detection in hyperspectral images according to macroscopic mixture modeling. For instance, a posteriori statistical tests have been used to understand bilinearity [4143].

\section{HYPERSPECTRAL MAPPING AT THE GLOBAL SCALE?}

One of the most important lines of research in urban remote sensing is nowadays urban monitoring at the national, continental or global scale. There are currently groups working on multispectral data, thanks to the Landsat legacy and the newly and freely available Sentinel data [44], and groups working on radar data, thanks to its all-weather capability, and finer spatial resolution [45]. Very small attention has been paid to spaceborne hyperspectral data [46, 47], essentially because they are geographically very sparse. The opposite constrains of a limited storage space on-board and a spatial resolution as fine as the corresponding multispectral sensors lead usually to on-demand acquisitions. Still, this scenario does not prevent from using hyperspectral data to map urban areas whenever they are available, complementing in time and/or space other existing data sets. To this aim, a methodology to map human settlements (meaning both built-up areas and other artificial surfaces) still needs to be designed, in order to work at every latitude, season of the year, and acquisition geometry.

\section{A. Urban extent extraction via urban fraction unmixing}

Urban textures in hyperspectral images with spatial resolutions coarser than a single urban object are characterized by strong nonlinear contributions. Hence, the results of nonlinear unmixing applied to hyperspectral imagery can be combined into a metric in order to map and monitor urban extents. According to what has been mentioned in the previous section, the methods in [10] and [40] are good candidates in this sense, as they provide reliable evaluations of nonlinear phenomena in urban areas. Indeed, in [11] the volumetric abundances obtained by the polytope decomposition (POD) algorithm in [40] are used to compute a urbanization index. This index is defined as the complement to the fraction of the total abundances due to natural materials in the scene, whose endmembers (differently from artificial ones) are easily known in advance. Experimental validations in different geographical locations proved that anthropogenic extents are better characterized by the POD algorithm than by any other linear or bi-linear models, and non-urban regions are properly separated from artificial surfaces. Accordingly, POD-based segmentation of urban areas outperforms those obtained by other models and better match a visual interpretation of the same scenes.

\section{CONClusions AND FUtURE DiRECTIONS FOR RESEARCH}

While this is a review paper, a few conclusions can be drawn. Interesting lines of research remain still open, and we look forward to reading exciting works by colleagues widening the range of urban applications of hyperspectral imaging. The points that we would like to stress as preliminary results of this review are therefore as follows:

1) The large variability (both in the spectral and spatial domain) of human settlements calls for more tests, by far larger of the existing data sets. Hyperspectral imaging in its infancy stage has focused mostly on natural environments (e.g. geology), as they served the purpose of highlighting the advantages of this technology. However, the role of human settlements for climate change is not fully understood. It requires more physical based information about object materials and their configuration in the urban environment to analyze the link between these characteristics and urban ecosystem functions. Hyperspectral imaging plays an essential role here.

2) So far, the huge potential of using the hyper-dimensional spectral feature space for analyzing texture and structure metrics of the urban environment is still not used and calls for further algorithms development. Moreover, the knowledge about the scale dependency of spectral and textural information can largely contribute to use spaceborne hyperspectral sensors despite their rather coarse spatial resolution between $20 \mathrm{~m}$ to $30 \mathrm{~m}$.

3) Very few studies are available that consider future spaceborne hyperspectral systems such as EnMAP, PRISMA, and HyspIRI. For global scale analyses of the urban environment, this has to be intensified, since the transferability of algorithms developed on VHR scale to coarser scales required different strategies and theories. The previously mentioned points can greatly contribute and prepare the ground for a global human settlement and infrastructure monitoring.

\section{REFERENCES}

[1] W. Heldens, U. Heiden, T. Esch, E. Stein and A.s Müller, "Can the Future EnMAP Mission Contribute to Urban Applications? A Literature Survey," Remote Sensing, vol. 3, no. 9, pp. 1817-1846, 2011.

[2] Heiden, U., Heldens, W., Roessner, S., Segl, K., Esch, T., Mueller, A. (2012): Urban structure type characterization using hyperspectral remote sensing and height information, Landscape and Urban Planning 105(4): 361-375.

[3] Herold, M., Roberts, D.A. (2005): Spectral characteristics of asphalt road aging and deterio-ration: implications for remote-sensing applications, Appl Opt 44(20): 4327-4334.

[4] F. Frassy, G. Candiani, M. Rusmini, P. Maianti, A. Marchesi, F. Rota Nodari, G. Dalla Via, C. Albonico and M. Gianinetto, "Mapping asbestos-cement roofing with hyperspectral remote sensing over a large mountain region of the Italian Western Alps", Sensors, vol. 14, pp. 15900-15913, 2014.

[5] Alonzo, M., Bookhagen, B., Roberts, D. (2014): Urban tree species mapping using hyper-spectral and lidar data fusion, Remote Sensing of Environment 148: 70-83.

[6] F. Dell'Acqua, P. Gamba, A. Ferrari, J. A. Palmason, J. A. Benediktsson and K. Arnason, "Exploiting spectral and spatial information in hyperspectral urban data with high resolution," IEEE Geoscience Remote Sensing Lett., vol. 1, no. 4, pp. 322-326, 2004.

[7] Behling, R., Bochow, M., Förster, S., Roessner, S., Kaufmann, H. 
(2015): Automated GIS-based derivation of urban ecological indicators using hyperspectral remote sensing and height information, Ecological Indicators 48: 218-234

[8] Heldens, W., Heiden, U., Esch, T., Mueller, A., Dech, S. (2017): Integration of remote sensing based surface information into a threedimensional microclimate model. ISPRS Journal of Photogrammetry and Remote Sensing 125: 106-124

[9] Roessner, S., Segl, K., Heiden, U., Kaufmann, H. (2001): Automated differentiation of urban surfaces based on airborne hyperspectral imagery, IEEE Transactions on Geoscience and Remote Sensing 39(7): $1525-1532$.

[10] A. Marinoni, A. Plaza and P. Gamba, "Harmonic Mixture Modeling for Efficient Nonlinear Hyperspectral Unmixing," IEEE J. Selected Topics in Applied Earth Obs. Remote Sens., vol. 9, no. 9, pp. 4247-4256, 2016.

[11] A. Marinoni and P. Gamba, "Accurate Detection of Anthropogenic Settlements in Hyperspectral Images by Higher Order Nonlinear Unmixing," IEEE J. Selected Topics in Applied Earth Obs. Remote Sens., vol. 9, no. 5, pp. 1792-1801, 2016.

[12] Herold, M., Roberts, D.A., Gardner, M.E., Dennison, P.E. (2004): Spectrometry for urban area remote sensing-Development and analysis of a spectral library from 350 to $2400 \mathrm{~nm}$, Remote Sensing of Environment 91: 304-319.

[13] Heiden, U., Segl, K., Roessner, S. and Kaufmann, H. (2007): Determination of robust spectral features for identification of urban surface materials in hyperspectral remote sensing data, Remote Sensing of Environment, 111: 537-552.

[14] Anderson, J.R., Hardy, E.E., Roach, J.T. and Witmer, R.E. (1976): A Land Use and Land Cover Classification Scheme for Use with Remote Sensor Data, U.S. Geological Survey.

[15] Ben-Dor, E., Levin, N., Saaroni, H. (2001): A spectral based recognition of the urban environment using the visible and near-infrared spectral region $(0.4-1.1 \mu \mathrm{m})$. A case study over Tel-Aviv, Israel, International Journal of Remote Sensing, 22(11): 2193-2218.

[16] Lachérade, S., Miesch, C., Briottet, X., Le Men, H. (2005): Spectral variability and bidirectional reflectance behaviour of urban materials at a $20 \mathrm{~cm}$ spatial resolution in the visible and near infrared wavelengths. A case study over Toulouse (France), Int J Remote Sens 26: 3859-3866.

[17] Meister, G., Rothkirch, A., Spitzer, H., Bienlein, J. (2000): BRDF field studies for remote sensing of urban areas, Remote Sens Rev 19: 37-57.

[18] Lachérade, S., Miesch, C., Boldo, D., Briottet, X., Valorge, C., Men, H.L. (2008): ICARE: A physically-based model to correct atmospheric and geometric effects from high spatial and spectral remote sensing images over 3D urban areas, Meteorol Atmos Phys 102(3): 209-222.

[19] Kotthaus, S., Smith, T.E.L., Wooster, M. J., Grimmond, C.S.B. (2014): Derivation of an urban materials spectral library through emittance and reflectance spectroscopy, ISPRS Journal of Photogrammetry and Remote Sensing 94: 194-212.

[20] Alonzo, M., McFadden, J.P., Nowak, D.J., Roberts, D.A. (2016): Mapping urban forest struc-ture and function using hyperspectral imagery and lidar data, Urban Forestry \& Urban Green-ing 17: 135-147.

[21] Escobedo, F.J., Nowak, D.J. (2009). Spatial heterogeneity and air pollution removal by an urban forest. Landscape Urban Plann. 90(3-4): $102-110$

[22] Manning, W.J. (2008): Plants in urban ecosystems: essential role of urban forests inurban metabolism and succession toward sustainability, Int. J. Sust. Dev. World Ecol. 15(4), 362-370.

[23] Lacan, I., McBride, J.R. (2008): Pest Vulnerability Matrix (PVM): a graphic model for assessing the interaction between tree species diversity and urban forest susceptibility to insects and diseases, Urban For. Urban Green 7(4): 291-300.

[24] Alonzo, M., Roth, K.L., Roberts, D.A. (2013): Identifying Santa Barbara's urban tree species from AVIRIS imagery using canonical discriminant analysis. Remote Sens. Lett. 4(5), 513-521.

[25] Artigas, F., Pechmann, I.C. (2010): Balloon imagery verification of remotely sensed Phragmites australis expansion in an urban estuary of New Jersey, USA, Landscape and Urban Planning 95(3): 105-112.

[26] Zhang, C., Qiu, F. (2012): Mapping individual tree species in an urban forest usingairborne lidar data and hyperspectral imagery. Photogramm. Eng. Remote Sens. 78(10): 1079-1087.

[27] Gu, H., Singh, A., Townsend, P.A. (2015): Detection of gradients of forest composition in an urban area using imaging spectroscopy, Remote Sensing of Environment 167: 168-180.

[28] Brackx, M., van Wittenberghe, S., Verhelst, J., Scheunders, P., Samson, R. (2017): Hyperspectral leaf reflectance of Carpinus betulus L. saplings for urban air quality estimation, Environmental Pollution 220: 159-167.
[29] Delegido, J., van Wittenberghe, S., Verrelst, J., Ortiz, V., Veroustraete, F., Valcke, R., Samson, R., Rivera, J.P., Tenjo, C., Moreno, J. (2014): Chlorophyll content mapping of urban vegetation in the city of Valencia based on the hyperspectral NAOC index, Ecological Indicators 40: 34 42.

[30] van Wittenberghe, S., Alonso, L., Verrelst, J., Hermans, I., Delegido, J., Veroustraete, F., Valcke, R., Moreno, J., Samson, R. (2013): Upward and downward solar-induced chlorophyll fluorescence yield indices of four tree species as indicators of traffic pollution in Valencia, Environmental Pollution 173: 29-37.

[31] Powell RL, Roberts DA, Dennison PE, Hess LL (2007) Sub-pixel mapping of urban land cover using multiple endmember spectral mixture analysis: Manaus, Brazil. Remote Sensing of Environment ,106: 253267.

[32] Roberts DA, Batista G, Pereira J, Waller E, Nelson B (1999) Change Identification Using Multitemporal Spectral Mixture Analysis: Applications in Eastern Amazonia. In: Lunetta R, Elvidge C, editors. London: Taylor \& Francis. pp. 318, 16.

[33] Liu T, Yang XJ (2013) Mapping vegetation in an urban area with stratified classification and multiple endmember spectral mixture analysis. Remote Sensing of Environment, 133: 251-264.

[34] A. Marinoni, A. Plaza and P. Gamba, "A Novel Pre-unmixing Framework for Efficient Detection of Linear Mixtures in Hyperspectral Images," IEEE Trans. Geoscience Remote Sens., vol. 55, no. 8, pp. 4325-4333, 2017.

[35] A. Halimi, Y. Altmann, N. Dobigeon, and J.-Y. Tourneret, "Nonlinear unmixing of hyperspectral images using a generalized bilinear model," IEEE Trans. Geosci. Remote Sens., vol. 49, no. 11, pp. 4153-4162, Nov. 2011.

[36] R. Heylen, M. Parente, and P. Gader, "A review of nonlinear hyperspectral unmixing methods", IEEE J. Sel. Top. App. Earth Obs. Rem. Sens., vol. 7, no. 6, pp. 1844-1868, June 2014.

[37] R. Heylen and P. Scheunders. "A multilinear mixing model for nonlinear spectral unmixing", IEEE Trans. Geosci. Remote Sens., vol. 54, no. 1, pp. 240-251, Jan 2016.

[38] R. Ammanouil, A.Ferrari, C. Richard, and S. Mathieu, "Nonlinear unmixing of hyperspectral data with vector-valued kernel functions", IEEE Trans. on Image Proc., vol. 26, no. 1, pp. 340-354, Jan. 2017.

[39] A. Halimi, P. Honeine, and J.M. Bioucas-Dias, "Hyperspectral unmixing in presence of endmember variability, nonlinearity, or mismodeling effects" IEEE Trans. on Image Proc., vol. 25, no. 10, pp. 4565-4579, Oct. 2016

[40] A. Marinoni, and P. Gamba, "A Novel Approach for Efficient p-linear Hyperspectral Unmixing", IEEE J. of Selected Topics in Signal Processing, vol. 9, no. 6, pp. 1156-1168, 2015.

[41] Y. Altmann et al. "Nonlinearity detection in hyperspectral images using a polynomial post-nonlinear mixing model," IEEE Trans. on Image Proc., vol. 22. No. 4, pp. 1267-1274, Apr. 2013.

[42] Y.Altmann et al., "Residual component analysis of hyperspectral images- application to joint nonlinear unmixing and nonlinearity detection", IEEE Trans. on Image Proc., vol.23, no. 5, pp. 2148-2158, May 2014.

[43] Y. Altmann et al., "Unsupervised post-nonlinear unmixing of hyperspectral images using a Hamiltonian Monte Carlo algorithm", IEEE Trans. on Image Proc., vol. 23, mo. 6, pp. 2663-2675, June 2014.

[44] M. Pesaresi, D. Ehrlich, S. Ferri, A. Florczyk, S. Freire, M. Halkia. Julea, T. Kemper, P. Soille, V. Syrris, "Operating Procedure for the Production of the Global Human Settlement Layer from Landsat Data of the Epochs 1975, 1990, 2000, and 2014", JRC Technical Report, European Commission, Joint Research Centre, Institute for the Protection and Security of the Citizen, Ispra (Italy), 2016.

[45] M. Marconcini, T. Esch, A. Felbier, W. Heldens, "Unsupervised highresolution global monitoring of urban settlements", Proceedings of the 2013 IEEE International Geoscience and Remote Sensing Symposium, Melbourne, Australia, 21-26 July 2013, pp. 4241-4244.

[46] Okujeni, A., van der Linden, S., Hostert, P. (2015): Extending the vegetation-impervious-soil model using simulated EnMAP data and machine learning. Remote Sensing of Environment 158: 69-80.

[47] M.Jilge, H.Feilhauer, U. Heiden, C, Juergens, "Concrete Meets Ecological Theory - A New Approach For Mapping Of Urban Surface Gaterial Gradients With EnMAP Data," EARSeL IS Workshop 2017 in Zurich, Switzerland, 2017. 